\title{
The end point of the first-order phase transition of the SU(2) gauge-Higgs model on a 4-dimensional isotropic lattice
}

\author{
Y. Aoki ${ }^{1,2}$ F. Csikor, ${ }^{3}$ Z. Fodor, ${ }^{3}$ and A. Ukawa ${ }^{1,2}$ \\ ${ }^{1}$ Center for Computational Physics, University of Tsukuba, Tsukuba, Ibaraki 305-8577, Japan \\ ${ }^{2}$ Institute of Physics, University of Tsukuba, Tsukuba, Ibaraki 305-8571, Japan \\ ${ }^{3}$ Institute for Theoretical Physics, Eötvös University, H-1088 Budapest, Hungary
}

(Received 28 January 1999; published 24 May 1999)

\begin{abstract}
We study the first-order finite-temperature electroweak phase transition of the SU(2) gauge-Higgs model defined on a four-dimensional isotropic lattice with the temporal extension $N_{t}=2$. A finite-size scaling study of Lee-Yang zeros yields the value of the Higgs self-coupling of the end point at $\lambda_{c}=0.00116(16)$. An independent analysis of the Binder cumulant gives a consistent value for the end point. Combined with our zerotemperature measurement of Higgs and $W$ boson masses, this leads to $M_{H, c}=73.3 \pm 6.4 \mathrm{GeV}$ for the critical Higgs boson mass beyond which the electroweak transition turns into a crossover. [S0556-2821(99)01311-9]

PACS number(s): 12.15.-y, 11.10.Wx, 11.15.Ha
\end{abstract}

\section{INTRODUCTION}

The minimal standard model predicts that the electroweak interaction undergoes a first-order phase transition at a finite temperature for light Higgs boson masses. A focus of recent studies has been whether the first-order phase transition survives with sufficient strength for a realistically heavy Higgs boson mass [1], since the feasibility of electroweak baryogenesis [2] depends crucially on it.

The first-order nature of the electroweak transition for light Higgs bosons can be shown within perturbation theory. However, perturbation theory breaks down for Higgs boson masses larger than about $M_{W}$ due to the bad infrared behavior of the gauge-Higgs part of electroweak theory [3]. Hence, numerical simulation techniques are needed to analyze the nature of the transition for heavy Higgs bosons.

Extensive studies in this direction have already been performed within the effective three-dimensional theory approach, in which all nonstatic modes of the system are integrated out perturbatively. This approach has the advantage that the full standard model including fermions can be mapped onto a three-dimensional $\mathrm{SU}(2)$ [or $\mathrm{SU}(2) \otimes \mathrm{U}(1)]$ gauge-Higgs model, as there are no fermionic static modes at finite temperature. In addition, thinning out the degree of freedom to those of a three-dimensional theory significantly reduces the computational requirement.

Results from simulations in this approach show that the first-order electroweak transition weakens as the Higgs boson mass increases [4-6], and that it turns into a continuous crossover for heavy Higgs bosons with a mass $M_{H} \gtrsim M_{W}[7]$. Detailed studies of the end point of the first-order transition including its universality class have also been made [8-10].

A potential problem with the three-dimensional approach is that it relies on perturbation theory to derive the threedimensional action so that numerical predictions may involve systematic errors due to truncation of perturbative series. From this point of view a direct simulation of the fourdimensional system is preferred. Results from fourdimensional simulations provide a check on those of the three-dimensional method.

Early studies of the four-dimensional SU(2) gauge-Higgs system were carried out in Refs. [11-15]. More recently ad- vances have been made with the use of the space-time anisotropic lattice $[16,17]$. This approach alleviates the doublescale problem that there are light modes with long wavelength, $\xi \gg 1 / T$, near the end point where the transition is of second order.

In this paper we report on a study of the end point of the SU(2) gauge-Higgs model employing four-dimensional space-time symmetric lattices with the temporal lattice size $N_{t}=2$, building upon a previous work [15]. Simulations have been carried out for a wide range of spatial lattice sizes, and finite-size scaling study of Lee-Yang zeros is used to find the location of the end point. We measure the Higgs and $W$ boson masses around the end point and estimate the value of the Higgs boson mass at the end point.

This paper is organized as follows. In Sec. II we present the SU(2) gauge-Higgs model lattice action and outline our strategy for finding the end point through Lee-Yang zeros. In Sec. III, following a brief discussion of susceptibility analysis, Lee-Yang zeros are examined. Another approach to finding the end point using the Binder cumulant is also described. In Sec. IV we present results of the zero-temperature mass measurement. Together with our result for the scalar self-coupling constant at the end point obtained through LeeYang zero analysis, this leads to the value of the Higgs boson mass at the end point. Section $\mathrm{V}$ is devoted to conclusions.

\section{THEORY AND SIMULATION}

We work with the standard SU(2) gauge-Higgs model action given by

$$
\begin{aligned}
S= & \sum_{x}\left[\sum_{\mu>\nu} \frac{\beta}{2} \operatorname{Tr} U_{x, \mu \nu}+\sum_{\mu} 2 \kappa L_{x, \mu}\right. \\
& \left.-\rho_{x}^{2}-\lambda\left(\rho_{x}^{2}-1\right)^{2}\right], \\
L_{x, \mu} \equiv & \frac{1}{2} \operatorname{Tr}\left(\Phi_{x}^{\dagger} U_{x, \mu} \Phi_{x+\hat{\mu}}\right), \quad \rho_{x}^{2} \equiv \frac{1}{2} \operatorname{Tr}\left(\Phi_{x}^{\dagger} \Phi_{x}\right),
\end{aligned}
$$


where $U_{x, \mu \nu}$ is the product of link operators around a plaquette, $\beta$ is related to the tree-level gauge coupling as $\beta$ $=4 / g^{2}, \kappa$ represents the Higgs field hopping parameter, and $\lambda$ is the scalar self-coupling. We put the system on a spacetime isotropic lattice of a size $N_{t} \times N_{s}^{3}$.

Finding the end point of the first-order finite-temperature phase transition of the model requires finite-size scaling analyses to quantitatively distinguish the case of a first-order transition from that of a crossover as the coupling parameters of the model are varied. As the main tool, we employ finitesize scaling analysis of Lee-Yang zeros $[18,19]$ on the complex $\kappa$ plane for fixed $\beta$ and $\lambda[8,9,17]$. For a first-order phase transition, the infinite volume limit of the zeros pinches the real $\kappa$ axis, while they stay away from it if there is no phase transition. We also supplement this method with analyses of susceptibility and Binder cumulant.

Our finite-temperature simulations are carried out for the temporal lattice size $N_{t}=2$. For the spatial lattice size we take $N_{s}^{3}=20^{3}, 24^{3}, 32^{3}, 40^{3}, 50^{3}$, and $60^{3}$. The gauge coupling is fixed at $\beta=8$. For the scalar self-coupling we choose five values, $\lambda=0.00075,0.001,0.00135,0.00145$, and 0.0017235 , which covers the range of the zero-temperature Higgs boson mass $57 \lesssim M_{H} \lesssim 85 \mathrm{GeV}$ [15]. For each value of $\lambda$ the scalar hopping parameter $\kappa$ is tuned to the vicinity of the pseudocritical point estimated by the peak position of the susceptibility of the Higgs field length squared $\rho^{2}$.

The updating algorithm is a combination of overrelaxation and heatbath methods [13], with the ratio of the two for the scalar part and the gauge part as specified in Ref. [15]. We make at least $10^{5}$ iterations of this hybrid overrelaxation algorithm at each coupling parameter point for each lattice size. The list of coupling values and statistics we use in our finite-temperature simulations are listed in Table I.

We also carry out zero-temperature simulations to measure the masses of Higgs and $W$ bosons around the end point of the first-order phase transition. For these runs an improved algorithm of Ref. [20] is employed. Details of the runs and results are discussed in Sec. IV.

\section{FINITE-TEMPERATURE RESULTS}

\section{A. Susceptibility}

Let us first look at the susceptibility of squared Higgs length,

$$
\chi_{\rho^{2}} \equiv V\left(\left\langle\rho^{2}\right\rangle-\langle\rho\rangle^{2}\right),
$$

where $V \equiv N_{s}^{3}$. The maximum value of the susceptibility at its peak, calculated by the standard reweighting technique [21] as a function of $\kappa$, is plotted in Fig. 1 against the spatial volume normalized by the critical temperature $V T_{c}^{3}$ $=N_{s}^{3} / N_{t}^{3}$. Errors are estimated by the jackknife procedure with the bin size of $10^{3}-10^{4}$ sweeps, which is listed in Table I.

The slope for the smallest scalar coupling $\lambda=0.00075$ approaches unity for large volumes, which is consistent with a first-order transition, while that for the largest coupling $\lambda$ $=0.0017235$ tends to a constant, showing an absence of a phase transition. A continuous decrease of the slope for the intermediate values of $\lambda$ indicates that the end point of the first-order transition is located in between the two extreme values. Our range of spatial volumes, unfortunately, is not sufficient to pin down the critical value of $\lambda$ from the susceptibility data.

\section{B. Lee-Yang zeros}

The determination of the end point of the finitetemperature phase transition of the model, thus a characteristic feature of the phase diagram, is made by the use of the Lee-Yang zeros of the partition function $Z[18,19]$. Near the first-order phase-transition point the partition function reads

$$
Z=Z_{s}+Z_{b} \propto \exp \left(-V f_{s}\right)+\exp \left(-V f_{b}\right),
$$

where the indices $s(b)$ refer to the symmetric (Higgs) phase and $f$ stands for the free-energy densities. Near the phasetransition point we also have

$$
f_{b}=f_{s}+\alpha\left(\kappa-\kappa_{c}\right),
$$

since the free-energy density is continuous. One then obtains

$$
Z \propto \exp \left[-V\left(f_{s}+f_{b}\right) / 2\right] \cosh \left[-V \alpha\left(\kappa-\kappa_{c}\right) / 2\right],
$$

which shows that for complex $\kappa Z$ vanishes at

$$
\operatorname{Im}(\kappa)=2 \pi(n-1 / 2) /(V \alpha)
$$

for integer $n$. In case a first-order phase transition is present, these Lee-Yang zeros move to the real axis as the volume goes to infinity. If a phase transition is absent the Lee-Yang zeros stay away from the real $\kappa$ axis. Thus, the way the Lee-Yang zeros move in this limit is a good indicator for the presence or absence of a first-order phase transition.

Calculation of the partition function for complex values of $\kappa$ is made with the reweighting method [21] in both imaginary and real directions of $\kappa$. In those cases where we have two ensembles with the same value of $\lambda$ and $N_{s}$, but different $\kappa$, we combine the two runs by setting the magnitude of the two partition functions to be equal at the midpoint between the two $\kappa$ 's.

In Fig. 2 we show the absolute value of the partition function normalized by its value at the real axis on the complex $\kappa$ plane,

$$
Z_{\text {norm }}(\kappa) \equiv\left|\frac{Z(\operatorname{Re} \kappa, \operatorname{Im} \kappa)}{Z(\operatorname{Re} \kappa, 0)}\right|
$$

for $\lambda=0.00075$ and $N_{s}=60$. The contour line of this figure is shown in Fig. 3. We observe three zeros in this case, whose distance from the real axis is roughly in the ratio 1:3:5 as expected from Eq. (7) for a first-order transition.

Let us call the zero nearest to the real axis the first zero, and denote its location by $\kappa_{0}$. We search for the first zero by the Newton-Raphson method applied to the equation

$$
Z(\operatorname{Re} \kappa, \operatorname{Im} \kappa)=0,
$$

starting with an initial guess for $\kappa_{0}$ obtained from the contour plot of $Z_{\text {norm }}(\kappa)$. The error of $\kappa_{0}$ is estimated by the 
TABLE I. Run parameters of finite-temperature simulation and results of the first Lee-Yang zero. Data used for analysis of susceptibility and the Binder cumulant are marked with $\chi$ and $B$, respectively, in the last column.

\begin{tabular}{|c|c|c|c|c|c|c|c|}
\hline & & & $\left(\times 10^{3}\right.$ & sweep) & & & \\
\hline$\lambda$ & $N_{s}$ & $\kappa$ & Iteration & Bin size & $\operatorname{Re} \kappa_{0}$ & $\operatorname{Im} \kappa_{0}$ & Use \\
\hline 0.00075 & 20 & 0.129114 & 100 & 2 & $0.1291133(23)$ & $0.0000477(20)$ & $\chi, B$ \\
\hline & 24 & 0.129103 & 100 & 2 & $0.1291068(12)$ & $0.0000285(11)$ & $\chi, B$ \\
\hline & 32 & 0.129102 & 100 & 4 & $0.12910273(91)$ & $0.00001351(45)$ & $\chi, B$ \\
\hline & 40 & 0.129100 & 100 & 6.25 & $0.12910086(72)$ & $0.00000762(26)$ & $\chi, B$ \\
\hline & 50 & 0.129100 & 120 & 10 & $0.12910041(51)$ & $0.00000411(17)$ & $\chi, B$ \\
\hline & 60 & 0.129100 & 180 & 10 & $0.129100308(303)$ & $0.000002321(51)$ & $\chi, B$ \\
\hline 0.001 & 20 & 0.129340 & 100 & 2 & (1203472(15) & $00000605(16)$ & $B$ \\
\hline & 20 & 0.129350 & 100 & 2 & $0.12934 / 2(15)$ & $0.0000000(10)$ & $\chi$ \\
\hline & 24 & 0.129330 & 100 & 2 & $0.1293357(18)$ & $0.0000432(18)$ & $\chi, B$ \\
\hline & 32 & 0.129328 & 100 & 2 & $0.12933093(122)$ & $0.00002136(75)$ & $\chi, B$ \\
\hline & 40 & 0.129327 & 100 & 2.5 & $0.12932802(80)$ & $0.00001223(44)$ & $\chi, B$ \\
\hline & 50 & 0.129327 & 100 & 4 & $012932797(37)$ & $000000763(26)$ & $B$ \\
\hline & 50 & 0.129328 & 100 & 4 & $0.12932197(37)$ & & $\chi$ \\
\hline & 60 & 0.1293275 & 180 & 7.5 & $0.12932743(37)$ & $0.00000489(18)$ & $\chi, B$ \\
\hline 0.00135 & 20 & 0.129660 & 100 & 1 & $0.1296888(34)$ & $0.0001167(36)$ & $\chi, B$ \\
\hline & 24 & 0.129650 & 100 & 1 & $0.1296619(29)$ & $0.0000819(32)$ & $\chi, B$ \\
\hline & 32 & 0.129644 & 100 & 1 & $0.1296465(20)$ & $0.0000542(20)$ & $\chi, B$ \\
\hline & 40 & 0.129640 & 100 & 2 & $0.1296426(15)$ & $0.0000293(11)$ & $\chi, B$ \\
\hline & 50 & 0.129639 & 120 & 2.5 & $0.12963782(137)$ & $0.00002016(88)$ & $\chi, B$ \\
\hline & 60 & 0.129637 & 120 & 4 & $0.12963754(68)$ & $0.00001299(78)$ & $\chi, B$ \\
\hline 0.00145 & 20 & 0.129748 & 100 & 1 & $0.1297482(35)$ & $0.0000885(38)$ & $\chi, B$ \\
\hline & 24 & 0.129736 & 100 & 1 & $0.1297384(20)$ & $0.0000567(20)$ & $\chi, B$ \\
\hline & 32 & 0.129728 & 100 & 2 & $0.1297318(15)$ & $0.0000328(12)$ & $\chi, B$ \\
\hline & 40 & 0.129724 & 100 & 2 & $0.12972751(115)$ & $0.00002171(99)$ & $\chi, B$ \\
\hline & 50 & 0.129722 & 120 & 2 & $0.12972654(80)$ & $0.00001529(79)$ & $\chi, B$ \\
\hline & 60 & 0.129724 & 120 & 4 & $0.12972517(61)$ & $0.00001146(79)$ & $\chi, B$ \\
\hline 0.0017235 & 20 & 0.129980 & 100 & 1 & $01200875(20)$ & $00000051(19)$ & \\
\hline & 20 & 0.129990 & 100 & 1 & $0.12998 / 2(20)$ & $0.0000951(19)$ & $\chi, B$ \\
\hline & 24 & 0.129980 & 100 & 2.5 & $0.1299755(24)$ & $0.0000604(21)$ & $\chi, B$ \\
\hline & 32 & 0.129966 & 100 & 1 & $0.1299654(15)$ & $0.0000383(12)$ & $\chi, B$ \\
\hline & 40 & 0.129968 & 100 & 1 & $0.1299663(15)$ & $0.0000276(14)$ & $\chi, B$ \\
\hline & 50 & 0.129965 & 100 & 2 & 0 1299616(14) & $00000207(16)$ & $\chi, B$ \\
\hline & 50 & 0.129966 & 100 & 2 & & & \\
\hline & 60 & 0.129962 & 120 & 4 & $0.12996122(71)$ & $0.00001585(74)$ & $\chi, B$ \\
\hline
\end{tabular}

jackknife method with a bin size given in Table I, i.e., the zero search is repeated for the set of partition functions calculated from each jackknife sample of configurations, and the jackknife formula is applied to the set of $\kappa_{0}$. The results for $\kappa_{0}$ are given in Table I. We show in Fig. 4 values of the imaginary part of the first zero $\operatorname{Im} \kappa_{0}(V)$ as a function of inverse volume.

Finite-size scaling theory predicts that the volume dependence of the imaginary part of the first zero is given by a scaling form,

$$
\operatorname{Im} \kappa_{0}(V)=\kappa_{0}^{c}+C V^{-\nu}
$$

For a first-order phase transition, the infinite volume limit vanishes, $\kappa_{0}^{c}=0$, and the exponent takes the value $\nu=1$. In the absence of a phase transition, $\kappa_{0}^{c} \neq 0$ and the value of the exponent is generally unknown.

In Fig. 5 we plot results for $\kappa_{0}^{c}$ as a function of $\lambda$ obtained by fitting the volume dependence of the first zero by the form (10) (see Fig. 4 for fit lines). Both $\kappa_{0}^{c}$ and $\nu$ are taken as fit parameters, and the entire set of volume $N_{s}^{3}=20^{3}-60^{3}$ is employed. Filled symbols mean that they are directly obtained from the simulations carried out at the corresponding values of $\lambda$. The points plotted with open symbols are ob- 


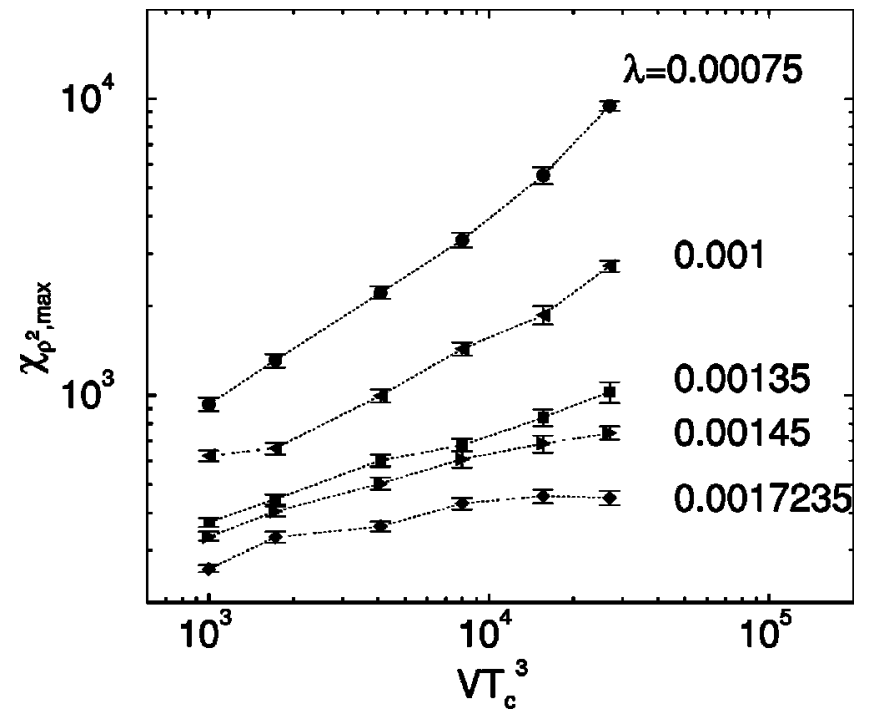

FIG. 1. Peak height of susceptibility of $\rho^{2}$ against inverse volume normalized by critical temperature $V T_{c}^{3}=N_{s}^{3} / N_{t}^{3}$. Dotted lines are guides for eyes.

tained from the first zero of the partition function calculated by reweighting the partition function measured at the point where $\kappa_{0}^{c}$ with the filled symbol of the same shape is shown. The agreement of open symbols of different shapes within errors shows that reweighting from different values of $\lambda$ gives consistent results between the measured points.

At small couplings $\lambda \lesssim 0.001, \kappa_{0}^{c}$ is consistent with zero, which agrees with the result of Ref. [15] that the transition is of first order in this region. At large couplings $\lambda$ $\gtrsim 0.0013, \kappa_{0}^{c}$ no longer vanishes, and hence there is no phase transition. In order to determine the end point of the phase transition, we take the three filled points at $\lambda$ $=0.00135,0.00145$, and 0.0017235 directly obtained from independent simulations without $\lambda$ reweighting, and make a fit with a function linear in $\lambda$. This gives the position of the end point to be

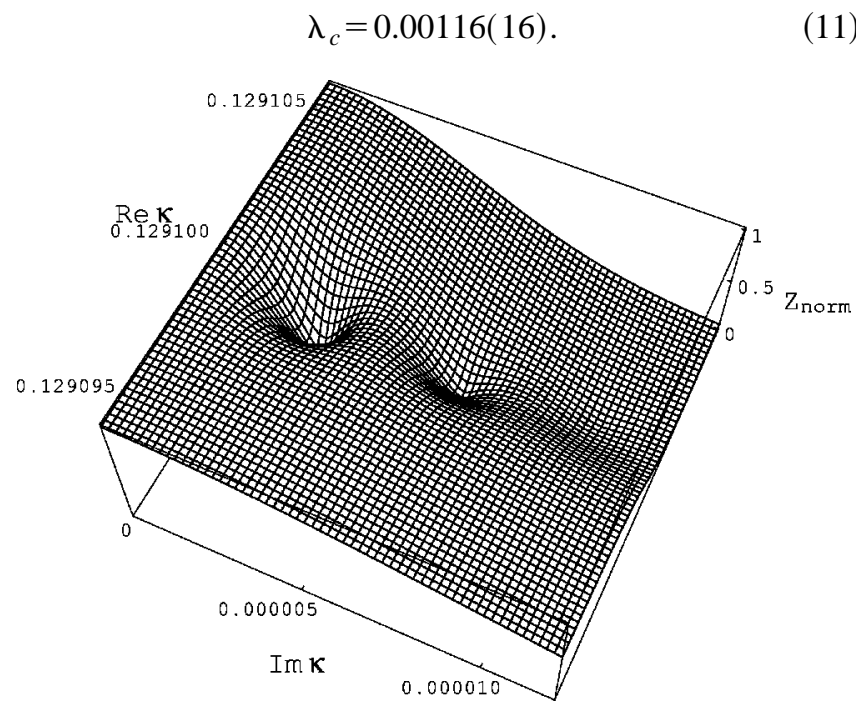

FIG. 2. Absolute value of normalized partition function as a function of complex $\kappa$ for $\lambda=0.00075$ and $N_{s}=60$.

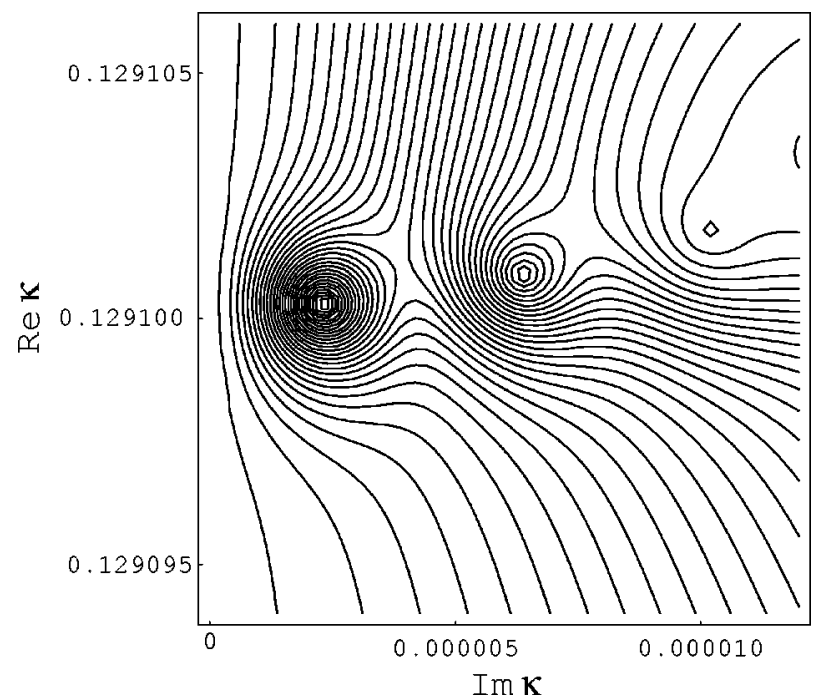

FIG. 3. Contour plot of Fig. 2.

In Fig. 6 we show the exponent of scaling function (10). The meanings of the symbols are the same as in Fig. 5. For $\lambda>\lambda_{c}$, where there is no phase transition, the exponent takes a value $\nu \approx 0.75$. Below the end point $\lambda<\lambda_{c}$, the exponent shows some trend of increase, but not quite to the value $\nu=1$ expected for a first-order transition. We think that this is due to insufficient volume sizes used in our simulation, for which corrections to the leading $1 / \mathrm{V}$ behavior are not negligible.

To check this point we make an alternative fit of results for the first zero adopting a quadratic ansatz in the volume given by

$$
\operatorname{Im} \kappa_{0}(V)=\kappa_{0}^{c}+C V^{-1}+D V^{-2}
$$

and show the results for $\kappa_{0}^{c}$ in Fig. 7. Clearly the infinite volume limit $\kappa_{0}^{c}$ starts to deviate from zero around $\lambda$

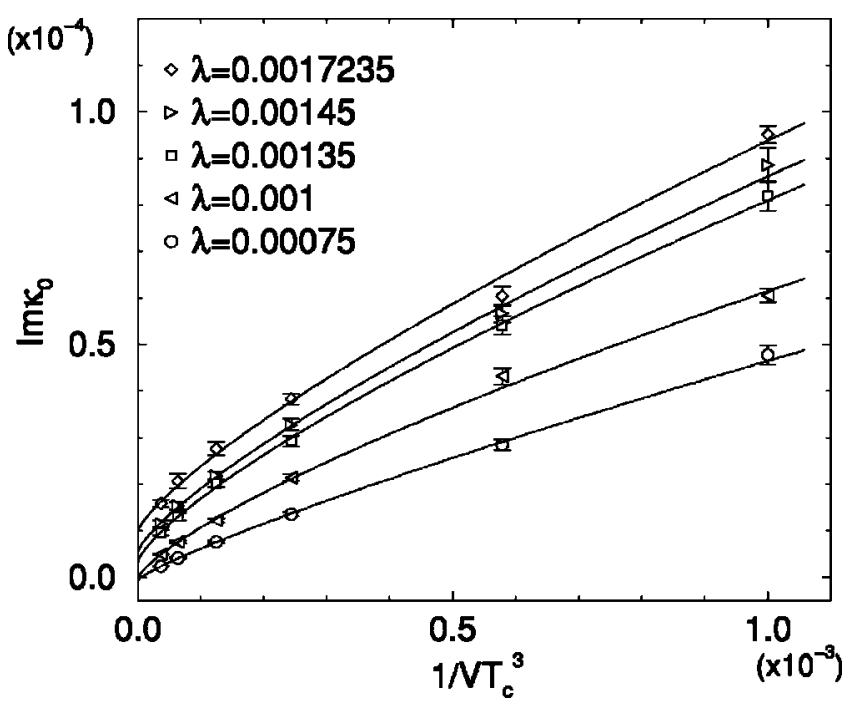

FIG. 4. Imaginary part of first Lee-Yang zero as a function of inverse volume normalized by the critical temperature. Solid lines are least $\chi^{2}$ fits with $\operatorname{Im} \kappa_{0}(V)=\kappa_{0}^{c}+C V^{-\nu}$. 


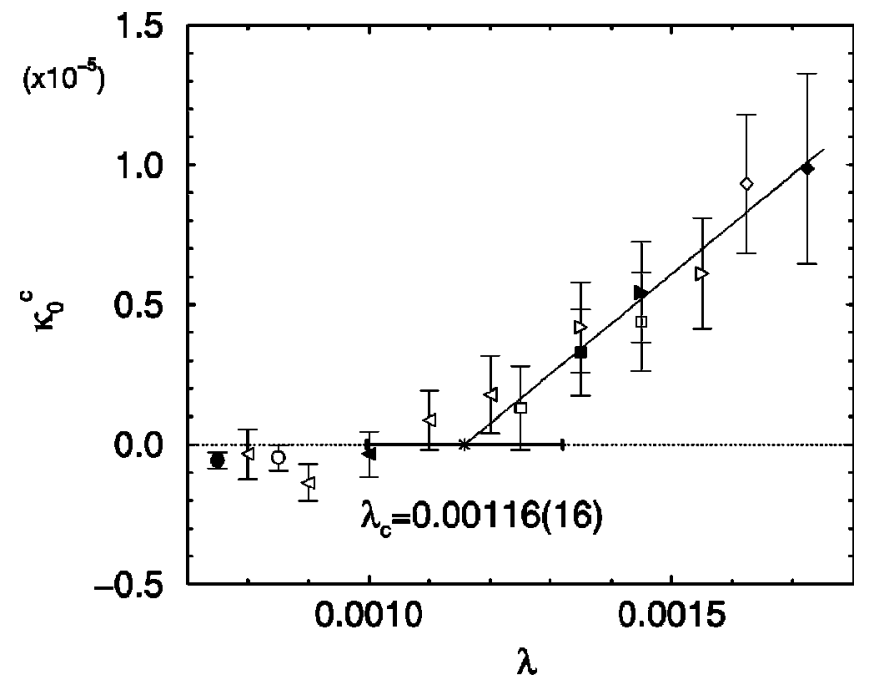

FIG. 5. Imaginary part of first Lee-Yang zero at infinite-volume limit as a function of Higgs self-coupling. Filled symbols are calculated without $\lambda$ reweighting, while open symbols with $\lambda$ reweighting from the filled symbol with same shape. Solid line is a linear fit to $\lambda=0.00135,0.00145$, and 0.0017235 (filled symbols).

$\approx 0.001$, which is consistent with the estimate of $\lambda_{c}$ above, albeit located at the lower end of the one standard deviation error band.

We note that the quadratic ansatz (12), formally the first three terms of a Laurent series, is expected to be correct in the case of a first-order phase transition, for which Eq. (7) describes the thermodynamic limit. However, it is not a valid assumption in the region of $\lambda$ where there is no phase transition. Therefore, unlike the case of Fig. 5, extrapolating the results of Fig. 7 from large to small values of $\lambda$ to estimate the location of the end point $\lambda_{c}$ is not justified.

\section{Binder cumulant}

Let us consider the Binder cumulant (cf. [22]) of the spacelike link operator,

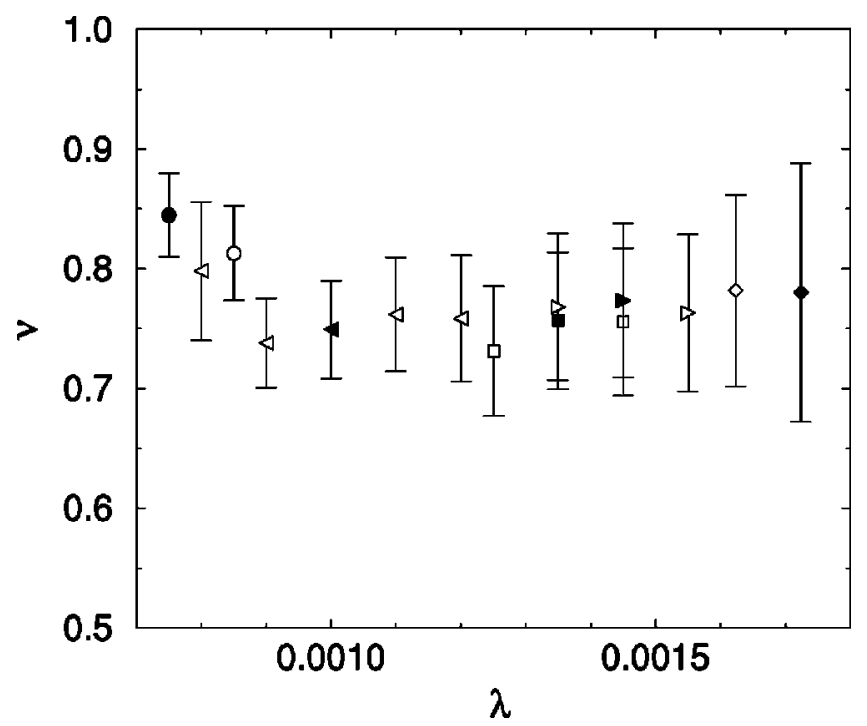

FIG. 6. Exponent $\nu$ of finite-size scaling of first Lee-Yang zero.

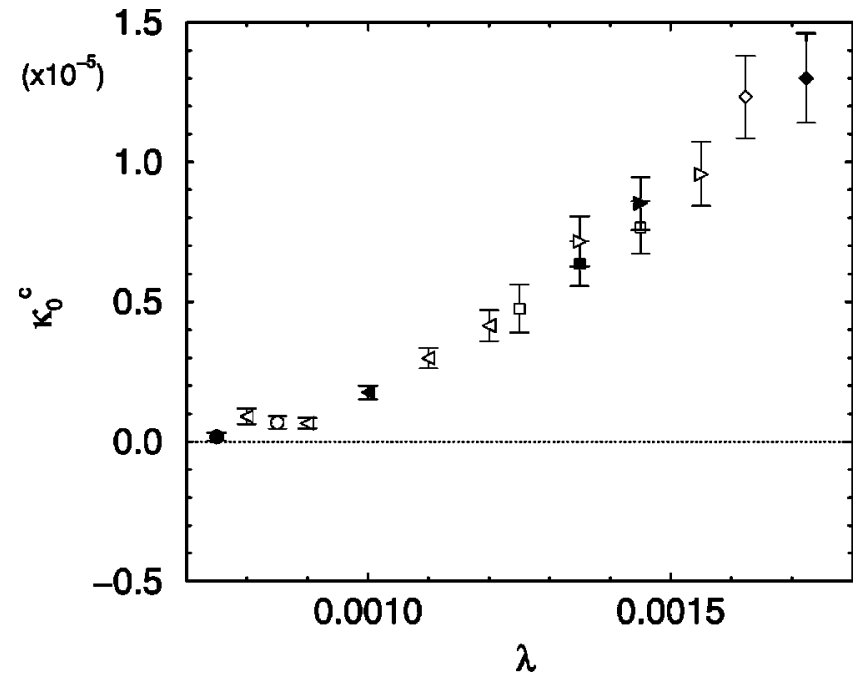

FIG. 7. Same as in Fig. 5. The quadratic polynomial is used for fit instead of the power function.

$$
B_{L_{s}}(\kappa) \equiv 1-\frac{\left\langle L_{s}^{4}\right\rangle}{3\left\langle L_{s}^{2}\right\rangle^{2}} ; \quad L_{s}=\frac{1}{3 N_{s}^{3} N_{t}} \sum_{x, \mu=1,2,3} L_{x, \mu}
$$

The infinite volume limit of the minimum of this quantity should deviate from $2 / 3$ for a first-order phase transition, while it should converge to $2 / 3$ beyond the endpoint.

We evaluate the minimum of the cumulant as a function of $\kappa$ for a given $\lambda$ and volume using reweighting. We then use a scaling ansatz

$$
B_{L_{s}}^{\min }=B_{L_{s}}^{c}+C V^{-\nu}
$$

to extract the infinite-volume value $B_{L_{s}}^{c}$.

In Fig. 8 we show $-\left(B_{L_{s}}^{c}-2 / 3\right)$ as a function of $\lambda$, where the meanings of the symbols are the same as in Fig. 5. A change of behavior from nonvanishing values to those consistent with zero at $\lambda \approx 0.001$ shows that the first-order phase

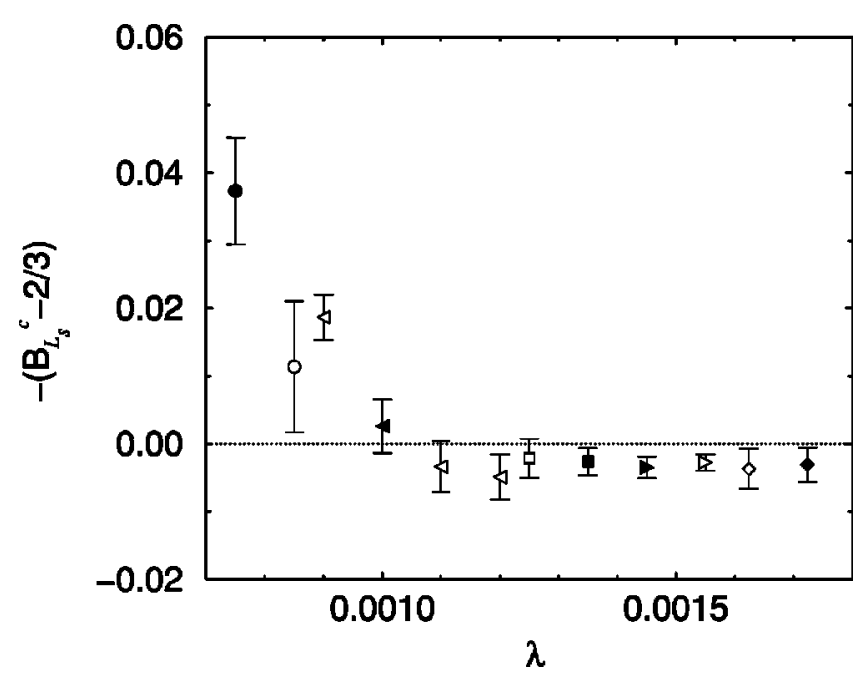

FIG. 8. Minimum value of Binder cumulant of $L_{s}$ at infinite volume limit as a function of $\lambda$. 
TABLE II. Run parameters of zero-temperature simulations and results for masses in lattice units.

\begin{tabular}{ccccc}
\hline \hline \multicolumn{1}{c}{$\lambda$} & \multicolumn{5}{c}{$\left(\times 10^{3}\right.$ sweep $)$} & & \\
\multicolumn{1}{c}{$\kappa$} & $N_{s}^{3} \times N_{t}$ & Iteration & $m_{H}$ & $m_{W}$ \\
\hline & $8^{3} \times 20$ & 60 & $0.2938(44)$ & $0.3583(41)$ \\
& $10^{3} \times 24$ & 75 & $0.2662(24)$ & $0.3380(33)$ \\
0.0011 & $12^{3} \times 28$ & 49 & $0.2844(46)$ & $0.3171(68)$ \\
0.129416 & $14^{3} \times 32$ & 34 & $0.2838(34)$ & $0.3191(69)$ \\
& $16^{3} \times 36$ & 26 & $0.2851(62)$ & $0.3152(133)$ \\
& $18^{3} \times 36$ & 26 & $0.2887(47)$ & $0.3321(100)$ \\
& & & & \\
0.00125 & $8^{3} \times 20$ & 60 & $0.2806(42)$ & $0.3285(95)$ \\
0.129532 & $10^{3} \times 24$ & 75 & $0.2764(33)$ & $0.3291(30)$ \\
& $14^{3} \times 32$ & 49 & $0.2884(38)$ & $0.2992(51)$ \\
& $16^{3} \times 36$ & 29 & $0.2851(56)$ & $0.3037(58)$ \\
& $18^{3} \times 36$ & 31.5 & $0.2863(91)$ & $0.2941(64)$ \\
\hline \hline
\end{tabular}

transition terminates around this value. Linearly extrapolating the two independent data at $\lambda=0.00075$ and 0.001 yields $\lambda_{c}=0.00102$ (3) for the end point, which is consistent with the result (11) from our study of Lee-Yang zeros. Note, however, that only two measured points are available for the linear extrapolation. Therefore, we cannot make a statement on the goodness of the fit. For this reason, we conservatively take the Lee-Yang value (11) as our best estimate of the end point.

\section{CRITICAL HIGGS BOSON MASS}

To determine the physical parameters characterizing the end point, namely the ratio of the Higgs boson mass to the $W$ boson mass and the renormalized gauge coupling $g_{R}$, we have to perform zero-temperature simulations. As in Refs. [12-14], we extract the Higgs boson mass $m_{H}$ in lattice units from correlators of $\rho_{x}^{2}$ and $L_{x, \mu}$. The $W$ boson mass in lattice units $m_{W}$ is obtained from the correlator of the composite link fields

$$
W_{x} \equiv \sum_{r, k=1}^{3} \frac{1}{2} \operatorname{Tr}\left(\tau_{r} \alpha_{x}^{\dagger} U_{x k} \alpha_{x+\hat{k}}\right)
$$

where $\tau_{r}$ is the Pauli matrix and $\alpha_{x}$ is the angle part of $\Phi_{x}$ such that $\Phi_{x} \equiv \rho_{x} \alpha_{x}$ with $\alpha_{x} \in \mathrm{SU}(2)$.

Masses are extracted from the correlators fitting to a hyperbolic cosine plus a constant function. Simple uncorrelated least-square fits and correlated fits with eigenvalue smoothing proposed by Michael and McKerrell [23] are used. The application of this method is discussed in detail in Ref. [14].

The actual procedure of extracting the mass parameters is the following. First we determine the reasonable time intervals for fitting the correlator data. The guideline is to choose as large an interval as possible with a reasonable $\chi^{2} /$ degree of freedom value. For this purpose correlated fits with eigenvalue smearing are used. We find this to be necessary since the data are strongly correlated for different time distances. Having fixed the fitting time interval, we next carry out un-

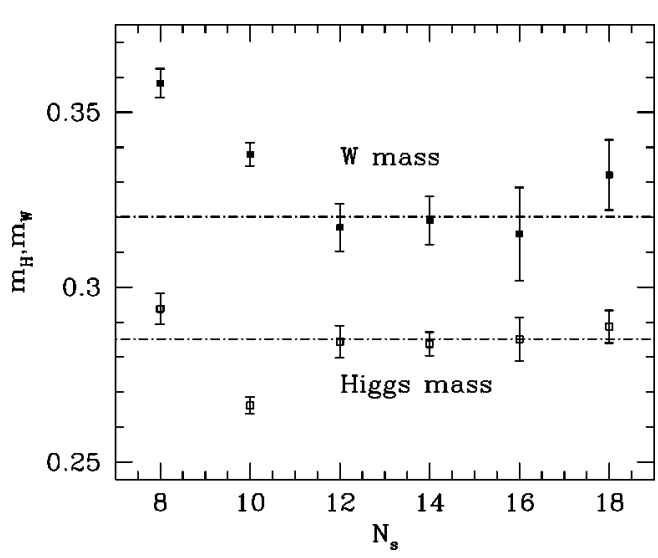

FIG. 9. Higgs and $W$ masses in lattice units as a function of $N_{s}$ for $\lambda=0.0011$

correlated fits. To perform this fit, we divide the data sample into subsamples, and estimate the errors of correlators from the statistical fluctuations of subsample averages.

The best fit value of the masses is taken to be the number given by the uncorrelated fit. The value of the Higgs boson mass is obtained by fitting to a linear combination of the two different correlators for $\rho_{x}^{2}$ and $L_{x, \mu}$. The errors on the masses are determined by jackknife analyses over subsamples. The masses obtained by the correlated fits with eigenvalue smearing are in all cases well within the error bars of the uncorrelated fits.

Our zero-temperature simulations are carried out at two points given by $\left[\lambda, \kappa=\kappa_{c}\left(\lambda, N_{t}=2\right)\right]$ for $\lambda=0.0011$ and 0.00125 employing several lattice sizes to examine finitevolume effects. The run parameters and results for masses are collected in Table II. The size of subsamples is typically 500 sweeps.

Our results do not show significant volume dependence (see Fig. 9), except for the two smallest spatial volumes $N_{s}$ $=8^{3}, 10^{3}$ for which somewhat different values are obtained compared to those of other volumes. We then discard those results and take an average over the rest of the volumes. This yields the values given in Table III. Setting $M_{W}=80 \mathrm{GeV}$, we obtain

$$
\begin{array}{cc}
M_{H}=70.9 \pm 1.1 \mathrm{GeV} & (\lambda=0.0011), \\
M_{H}=76.8 \pm 1.1 \mathrm{GeV} & (\lambda=0.00125) .
\end{array}
$$

Making a linear interpolation to the critical value $\lambda_{c}$ $=0.00116(16)$ from the Lee-Yang zero analysis, we find

TABLE III. Averaged masses in lattice units and renormalized gauge couplings from results in Table II excluding those for the two smallest volumes.

\begin{tabular}{lcccc}
\hline \hline \multicolumn{1}{c}{$\lambda$} & $m_{H}$ & $m_{W}$ & $R_{H W}$ & $g_{R}^{2}$ \\
\hline 0.0011 & $0.2852(22)$ & $0.3202(41)$ & $0.8864(136)$ & $0.5712(27)$ \\
0.00125 & $0.2877(26)$ & $0.2988(30)$ & $0.9607(134)$ & $0.5768(33)$ \\
\hline \hline
\end{tabular}


TABLE IV. Summary of the fit parameters for the static potential and the renormalized gauge coupling.

\begin{tabular}{|c|c|c|c|c|c|c|}
\hline $\begin{array}{l}\lambda \\
\kappa\end{array}$ & $N_{s}^{3} \times N_{t}$ & $A$ & $M$ & $D$ & $C$ & $g_{R}^{2} \equiv \frac{16}{3} \pi A$ \\
\hline & $12^{3} \times 28$ & $0.03495(58)$ & $0.3021(62)$ & $0.03941(68)$ & $0.0968(2)$ & $0.5856(97)$ \\
\hline 0.0011 & $14^{3} \times 32$ & $0.03435(52)$ & $0.2783(90)$ & $0.03673(45)$ & $0.09672(21)$ & $0.5755(87)$ \\
\hline \multirow[t]{3}{*}{0.129416} & $16^{3} \times 36$ & $0.03406(30)$ & $0.2898(107)$ & $0.03975(28)$ & $0.09632(13)$ & $0.5707(50)$ \\
\hline & $18^{3} \times 36$ & $0.03394(22)$ & $0.2791(42)$ & $0.04061(262)$ & $0.09633(3)$ & $0.5687(37)$ \\
\hline & $12^{3} \times 28$ & $0.03561(46)$ & $0.2788(121)$ & $0.02814(310)$ & $0.09751(31)$ & $0.5966(77)$ \\
\hline 0.00125 & $14^{3} \times 32$ & $0.03456(70)$ & $0.2573(113)$ & $0.0353(57)$ & $0.09766(39)$ & $0.5791(117)$ \\
\hline \multirow[t]{2}{*}{0.129532} & $16^{3} \times 36$ & $0.03386(31)$ & $0.2559(79)$ & $0.0416(35)$ & $0.09740(12)$ & $0.5673(52)$ \\
\hline & $18^{3} \times 36$ & $0.034442(35)$ & $0.2676(36)$ & $0.03831(41)$ & $0.09704(4)$ & $0.5770(59)$ \\
\hline
\end{tabular}

$$
M_{H, c}=73.3 \pm 6.4 \mathrm{GeV} \text {, }
$$

where the error is dominated by that of $\lambda_{c}$.

From measurements of Wilson loops we also determine the values of the renormalized gauge coupling $g_{R}$ using the method described in Refs. [12-14]. The potential as a function of the distance $R$ is fitted by

$$
V(R)=-\frac{A}{B} e^{-M R}+C+D G\left(M, R, L_{s}\right),
$$

where $G\left(M, R, L_{s}\right)$ stands for lattice artifacts (cf. [13]). The potential is determined from the rectangular Wilson loops by fitting the time dependence with three exponentials. A stable fit is obtained in all cases. The potential is then fitted by Eq. (19) using all $R$ values. Our results for the fit parameters and $g_{R}^{2}$ for various spatial size lattices are shown in Table IV. We see that $g_{R}$ is constant within errors. The averaged values are given in Table III. The values do agree within errors, showing that our simulations for the two $\lambda$ values correspond to the same renormalized gauge coupling. Therefore, the linear extrapolation to $\lambda_{c}$ mentioned above is justified, since we use Higgs boson masses at equal renormalized gauge couplings.

Finally, let us try to estimate the effect of fermions and the $U(1)$ gauge boson on our result. We make this estimation through the perturbative expression for the parameter $x=\lambda_{3} / g_{3}^{2}$ of the dimensionally reduced model in terms of the physical parameters of the standard model [24]. Using our results for the Higgs boson mass and the renormalized gauge coupling, we find $x_{c}=0.121 \pm 0.020$ for the end point. Including the effect of fermions and the $\mathrm{U}(1)$ gauge boson, this value corresponds to $M_{H, c}=80$ $\pm 7 \mathrm{GeV}$.

\section{CONCLUSIONS}

We have studied the end point of the finite-temperature first-order transition of the $\mathrm{SU}(2)$ gauge-Higgs model on a space-time isotropic lattice of a temporal extension $N_{t}=2$. The results from Lee-Yang zero and Binder cumulant analyses show that the first-order phase transition terminates at $\lambda_{c}=0.00116(16)$ and turns into a smooth crossover for $\lambda$ $>\lambda_{c}$.

Setting $M_{W}=80 \mathrm{GeV}$ our result for the critical Higgs boson mass is $M_{H, c}=73.3 \pm 6.4 \mathrm{GeV}$. This is consistent within error with the value $M_{H, c}=74.6 \pm 0.9 \mathrm{GeV}$ [17] obtained in a four-dimensional anisotropic lattice simulation for the same temporal size. The same work also reported that the critical mass decreases for larger temporal size, and extrapolates to $M_{H, c}=66.5 \pm 1.4 \mathrm{GeV}$ in the continuum limit. This value is consistent with the three-dimensional result $66.2 \mathrm{GeV}$ [9]. Thus results from various methods, in three and four dimensions, agree well.

For a comparison with the experimental lower bound $M_{H}>87.9 \mathrm{GeV}$ [25] for the Higgs boson mass, we need to include the effect of the fermions and U(1) gauge boson. The good agreement of critical mass from the fourand three-dimensional simulations noted above imply that this may be made perturbatively, with which we find $M_{H, c}=80 \pm 7 \mathrm{GeV}$ for our $N_{t}=2$ simulation. This value is about $10 \%$ larger, albeit with a comparable error, than the result $M_{H, c}=72.4 \pm 1.7 \mathrm{GeV}$ in the continuum limit obtained from a four-dimensional anisotropic study [17], possibly due to scaling violations. We also note that the three-dimensional approach reported the values $M_{H, c}=72.4 \pm 0.9 \mathrm{GeV}$ [9] and $M_{H, c}=72 \pm 2 \mathrm{GeV}$ [10]. Combining all the available results, we conclude that the electroweak baryogenesis within the minimal standard model is excluded.

\section{ACKNOWLEDGMENTS}

Part of this work was carried out while Z.F. was visiting KEK with support from the Foreign Researcher Program of the Ministry of Education of Japan. Part of the numerical calculations was made on the VPP-500/30 at the Information Processing Center of the University of Tsukuba and the PMS-11G PC-farm in Budapest. This work is supported in part by Grants-in-Aid from the Ministry of Education of Japan (Nos. 09304029, 10640246), Hungarian Science Foundation Grants No. OTKA-T016240/T022929, and Hungarian Ministry of Education Grant No. FKP-0128/ 1997. 
[1] For reviews, see, K. Rummukainen, in Lattice '96, Proceedings of the International Symposium, St. Louis, Missouri, edited by C. Bernard et al. [Nucl. Phys. B (Proc. Suppl.) 53, 30 (1997)]; M. Laine and K. Rummukainen, Lattice '98 [Nucl. Phys. B (Proc. Suppl.) (in press)], hep-lat/9809045.

[2] For a review, see, V. Rubakov and M. Shaposhnikov, Usp. Fiz. Nauk 166, 493 (1996) [Phys. Usp. 39, 461 (1996)].

[3] Z. Fodor and A. Hebecker, Nucl. Phys. B432, 127 (1994); W. Buchmüller, Z. Fodor, and A. Hebecker, ibid. B447, 317 (1995).

[4] K. Kajantie, K. Rummukainen, and M. Shaposhnikov, Nucl. Phys. B407, 356 (1993); K. Farakos, K. Kajantie, K. Rummukainen, and M. Shaposhnikov, Phys. Lett. B 336, 494 (1994).

[5] E.-M. Ilgenfritz, J. Kripfganz, H. Perlt, and A. Schiller, Phys. Lett. B 356, 561 (1995); M. Gürtler, E.-M. Ilgenfritz, J. Kripfganz, H. Perlt, and A. Schiller, Nucl. Phys. B (Proc. Suppl.) 49, 312 (1996); Nucl. Phys. B483, 383 (1997).

[6] F. Karsch, T. Neuhaus, A. Patkós, and J. Rank, Nucl. Phys. B474, 217 (1996).

[7] K. Kajantie, M. Laine, K. Rummukainen, and M. Shaposhnikov, Phys. Rev. Lett. 77, 2887 (1996); Nucl. Phys. B493, 413 (1997).

[8] F. Karsch, T. Neuhaus, A. Patkós, and J. Rank, Nucl. Phys. B (Proc. Suppl.) 53, 623 (1997).

[9] M. Gürtler, E.-M. Ilgenfritz, and A. Schiller, Phys. Rev. D 56, 3888 (1997).

[10] K. Rummukainen, M. Tsypin, K. Kajantie, M. Laine, and M. Shaposhnikov, Nucl. Phys. B532, 283 (1998).
[11] B. Bunk, E.-M. Ilgenfritz, J. Kripfganz, and A. Schiller, Phys. Lett. B 284, 371 (1992); B. Bunk, E.-M. Ilgenfritz, J. Kripfganz, and A. Schiller, Nucl. Phys. B403, 453 (1993).

[12] Z. Fodor, J. Hein, K. Jansen, A. Jaster, I. Montvay, and F. Csikor, Phys. Lett. B 334, 405 (1994).

[13] Z. Fodor, J. Hein, K. Jansen, A. Jaster, and I. Montvay, Nucl. Phys. B439, 147 (1995).

[14] F. Csikor, Z. Fodor, J. Hein, A. Jaster, and I. Montvay, Nucl. Phys. B474, 421 (1996).

[15] Y. Aoki, Phys. Rev. D 56, 3860 (1997).

[16] F. Csikor, Z. Fodor, and J. Heitger, Phys. Rev. D 58, 094504 (1998).

[17] F. Csikor, Z. Fodor, and J. Heitger, Phys. Rev. Lett. 82, 21 (1999).

[18] C. N. Yang and T. D. Lee, Phys. Rev. 87, 404 (1952); 87, 410 (1952).

[19] C. Itzykson, R. B. Pearson, and J. B. Zuber, Nucl. Phys. B220 [FS8], 415 (1983).

[20] B. Bunk, Nucl. Phys. B (Proc. Suppl.) 42, 566 (1995).

[21] I. R. McDonald and K. Singer, Discuss. Faraday Soc. 43, 40 (1967); A. M. Ferrenberg and R. Swendsen, Phys. Rev. Lett. 61, 2635 (1988); 63, 1195 (1989).

[22] K. Binder, Phys. Rev. Lett. 47, 693 (1981).

[23] C. Michael and A. McKerrell, Phys. Rev. D 51, 3745 (1995).

[24] K. Kajantie, M. Laine, K. Rummukainen, and M. Shaposhnikov, Nucl. Phys. B458, 90 (1996).

[25] ALEPH Collaboration, R. Barate et al., Phys. Lett. B 440, 403 (1998). 